\title{
Interplay between Ino80 and Swr1 chromatin remodeling enzymes regulates cell cycle checkpoint adaptation in response to DNA damage
}

\author{
Manolis Papamichos-Chronakis, ${ }^{1}$ Jocelyn E. Krebs, ${ }^{2}$ and Craig L. Peterson ${ }^{1,3}$ \\ ${ }^{1}$ Program in Molecular Medicine, University of Massachusetts Medical School, Worcester, Massachusetts 01605, USA; \\ ${ }^{2}$ Department of Biological Sciences, University of Alaska Anchorage, Anchorage, Alaska 99508, USA
}

\begin{abstract}
Ino80 and Swr1 are ATP-dependent chromatin remodeling enzymes that have been implicated in DNA repair. Here we show that Ino80 is required for cell cycle checkpoint adaptation in response to a persistent DNA double-strand break (DSB). The failure of cells lacking Ino80 to escape checkpoint arrest correlates with an inability to maintain high levels of histone $\mathrm{H} 2 \mathrm{AX}$ phosphorylation and an increased incorporation of the Htz1p histone variant into chromatin surrounding the DSB. Inactivation of Swr1 eliminates this DNA damage-induced Htz1p incorporation and restores $\mathrm{H} 2 \mathrm{AX}$ phosphorylation and checkpoint adaptation. We propose that Ino80 and Swr1 function antagonistically at chromatin surrounding a DSB, and that they regulate the incorporation of different histone $\mathrm{H} 2 \mathrm{~A}$ variants that can either promote or block cell cycle checkpoint adaptation.
\end{abstract}

[Keywords: Ino80; Swr1; Htz1; DNA repair; chromatin, checkpoint]

Supplemental material is available at http://www.genesdev.org.

Received April 12, 2006; revised version accepted July 7, 2006.

Chromosomal DNA double-strand breaks (DSBs) arise through exposure of cells to harmful environmental agents such as ionizing radiation or mutagenic chemicals. DSBs can also be caused by endogenously produced oxygen radicals, by errors in DNA replication, or as obligatory intermediates during programmed cellular processes (Paques and Haber 1999; Symington 2002; Peterson and Cote 2004). Cell survival and maintenance of genome integrity are dependent on efficient repair of DSBs, as unrepaired or misrepaired DSBs may lead to mutations, gene translocations, gross chromosomal rearrangements, or cellular lethality (Khanna and Jackson 2001).

Several pathways for repairing DSBs have evolved, and are highly conserved throughout eukaryotes. Homologous recombination (HR) is a major pathway of DSB repair in all eukaryotes that it is mostly error-free (Paques and Haber 1999; Symington 2002; Peterson and Cote 2004). A central hallmark of all types of homologous recombination reactions is the ability of the recombination machinery to use a single-stranded DNA (ssDNA) molecule, derived from processing of the DSB, to search

${ }^{3}$ Corresponding author.

E-MAIL craig.Peterson@umassmed.edu; FAX (508) 856-5011.

Article is online at http://www.genesdev.org/cgi/doi/10.1101/gad.1440206. for, capture, and invade a homologous DNA duplex. In contrast, an alternative pathway, nonhomologous end joining, involves the religation of the two broken ends by either an error-free or error-prone mechanism (Critchlow and Jackson 1998).

In eukaryotic cells, DNA damage causes a signal that arrests cell cycle progression at the G2/M boundary, allowing cells extra time to repair damage prior to segregation of chromosomes. This DNA damage checkpoint requires a group of highly conserved proteins, including yeast $\operatorname{Rad} 9 \mathrm{p}, \operatorname{Rad} 17 \mathrm{p}, \operatorname{Rad} 24 \mathrm{p}, \operatorname{Rad} 53 \mathrm{p}, \operatorname{Mec} 1 \mathrm{p}$, and Mec3p (Lydall and Weinert 1995). How these proteins detect and signal damage is not clear, nor is it known how the checkpoint is eliminated once the damage is repaired. In yeast, it has also been shown that cells can escape an extended checkpoint arrest and re-enter the cell cycle even with an unrepaired DSB (Sandell and Zakian 1993). This process has been termed checkpoint adaptation, and it may allow cells additional time to attempt DSB repair in the next cell division, or it has been proposed that the passage through mitosis with a DSB may activate a more efficient apoptotic-type pathway for removal of cells with persistent DNA damage (Lupardus and Cimprich 2005). Checkpoint adaptation was thought to be a process restricted to unicellular fungi, but a recent study has shown that adaptation also occurs in a vertebrate system (Yoo et al. 2005), and that at least 
some components of the pathway appear to be conserved. In yeast, several distinct genetic pathways have been identified that control this adaptation pathway. Each of these pathways is believed to function by downregulating the Rad53p checkpoint kinase, albeit by mostly unknown mechanisms (Pellicioli et al. 2001).

One of the initial events that occurs following formation of a DSB is the recruitment of an ATM/ATR kinase (Meclp/Tellp in yeast), which phosphorylates a C-terminal serine residue within the histone $\mathrm{H} 2 \mathrm{~A}$ variant, H2AX (Downs et al. 2000; Bassing et al. 2002; Redon et al. 2003). Histone $\mathrm{H} 2 \mathrm{AX}$ is a minor variant of core histone $\mathrm{H} 2 \mathrm{~A}$ that is present in mammalian chromatin at a frequency of approximately one $\mathrm{H} 2 \mathrm{AX}$ nucleosome per $10 \mathrm{H} 2 \mathrm{~A}$-containing nucleosomes. $\mathrm{H} 2 \mathrm{AX}$ is rapidly phosphorylated $\left(<5^{\prime}\right.$ following DSB formation) in a megabase chromatin domain surrounding chromosomal DSBs (Rogakou et al. 1999), and heterozygous or homozygous null alleles of mammalian $\mathrm{H} 2 \mathrm{AX}$ lead to an increased susceptibility to cancer and radiation sensitivity (Bassing et al. 2002; Celeste et al. 2002, 2003a). Likewise, yeast $\mathrm{H} 2 \mathrm{AX}$ (the major form of H2A in yeast) is phosphorylated within a $\sim 100-\mathrm{kb}$ domain of chromatin surrounding a single DSB (Shroff et al. 2004), and disruption of the phosphorylation site (S129) within H2AX causes mild sensitivity to a variety of DSB-inducing agents (Downs et al. 2000) (we will refer to yeast H2A as H2AX). Mechanistic studies in mammalian cells have shown that $\mathrm{H} 2 \mathrm{AX}$ phosphorylation is not required for initial recruitment of DNA repair factors to the DSB, but it does control their subsequent association into irradiation-induced foci (Celeste et al. 2003b). Recent studies in budding yeast have shown that H2AX-phos is required for recruitment of cohesin complex to a DSB which facilitates sister chromatid recombination (Strom et al. 2004; Unal et al. 2004).

The Saccharomyces cerevisiae INO80 and SWR1 genes encode DNA-stimulated ATPases that are the catalytic subunits of large, multisubunit chromatin remodeling complexes, Ino80 and Swr1, respectively (Shen et al. 2000; Mizuguchi et al. 2003). Genome-wide expression profiling has demonstrated that Ino80p and Swrlp are involved in transcriptional control (Shen et al. 2000; Mizuguchi et al. 2003; van Attikum et al. 2004), but several pieces of evidence implicate an independent role in DNA repair (Shen et al. 2000; Mizuguchi et al. 2003). First, ino80 and swr1 mutants are sensitive to several DNA damaging agents, including methylmethanesulfonate (MMS) and hydroxyurea (HU). These phenotypes do not appear to be due to a transcriptional defect, as the expression of DNA repair or cell cycle checkpoint factors are not affected by ino80 mutations, nor does an ino80 mutation affect the transcriptional response to DNA damage (Shen et al. 2000; van Attikum et al. 2004). Importantly, three recent reports have shown that Ino80 complex is recruited to a DSB, and this recruitment requires phosphorylation of histone H2AX (Downs et al. 2004; Morrison et al. 2004; van Attikum et al. 2004).

We investigated whether Ino80 contributes to repair of a DSB by either the homologous recombination or non- homologous end-joining pathways. Whereas Ino80 is mostly dispensable for both of these repair mechanisms, Ino80 is required for escape from an extended cell cycle checkpoint arrest (checkpoint adaptation). Furthermore, the absence of Ino80 leads to decreased levels of H2AX phosphorylation and increased incorporation of the Htzlp variant adjacent to a DSB. Inactivation of the Swr1 remodeling enzyme eliminates this aberrant Htzl incorporation and restores high levels of H2AX-phos and checkpoint adaptation in the absence of Ino80. The data suggest a model in which Ino80 and Swr1 catalyze a dynamic cycle of $\mathrm{H} 2 \mathrm{~A}$ variant exchange that controls whether to remove the checkpoint arrest and re-enter the cell cycle or to maintain arrest and eventually die.

\section{Results}

Although INO80 is required for resistance to genotoxic agents (Shen et al. 2000), it is not clear how Ino80p contributes to the mechanism of DNA DSB repair. Many of the in vivo analyses of DNA repair in yeast have exploited a GAL10-HO fusion gene that allows for the rapid, galactose-inducible formation of a single DSB at the MAT locus (White and Haber 1990). Recombinational repair of this HO-induced DSB can be monitored in strains that harbor the homologous HMRa or HML $\alpha$ donor sequences (a "switching" strain JKM154); or alternatively, repair of the HO-induced DSB by nonhomologous end-joining can be followed in strains where HMRa and HML $\alpha$ have been deleted ("donorless" strains; JKM179|. However, a previous study reported that a deletion allele of INO80 could not be recovered in the donorless JKM179 background, precluding further analysis of the role of Ino80p in repair of an HO-induced DSB (van Attikum et al. 2004). Likewise, Wu and colleagues concluded that an ino80s is also lethal in the w303 background (Shen et al. 2000). In contrast, an ino $80 \Delta$ has been successfully created in the s288c background, and in this case the ino80s strain shows a severe growth defect (Shen et al. 2000).

We noticed that some loci are generally refractory to deletion of the entire ORF by the one-step gene deletion strategy; and thus we worried that the inability to obtain an ino80s allele in some strain backgrounds may not be due to inviability but due instead to a problem with the recombination event itself. Consistent with this view, we were unable to delete the entire INO80 ORF even in a diploid strain of the w303 background (our unpublished results). In contrast, we were able to use the one-step gene deletion strategy to replace the first 900 base pairs (bp) of the INO80 ORF with either the KAN-MX6 gene or the TRP1 gene. In all cases, we were able to efficiently recover ino80 deletions in all strain backgrounds, including s288c, w303, and JKM179. Notably, all of these ino80s strains showed sensitivity to genotoxic agents, such as MMS and HU (Supplementary Fig. S1; data not shown), and our ino80s allele in the s288c background has a severe growth defect that is consistent with previous analyses of a complete ORF replacement (Shen et al. 
2000). Likewise, our N-terminal deletion allele causes an inositol auxotrophy (data not shown), a phenotype reported for a null allele (Ebbert et al. 1999). Our N-terminal deletion allele also appears to be ATPase defective, as the HU sensitivity of the ino80A::KAN-MX6 allele is complemented by a plasmid-borne copy of INO80 but not by an ATPase-defective allele (INO80-K737A) (Supplementary Fig. S1). Thus, our ino80s appears to be a null allele, suggesting that INO80 is unlikely to be an essential gene in commonly used yeast strain backgrounds. These results are consistent with the viability of gene deletions that remove other subunits of the Ino80 remodeling complex (van Attikum et al. 2004).

To investigate the functional role of Ino80 in repair of DSBs, we investigated whether inactivation of Ino80 leads to a defect in either nonhomologous end-joining or recombinational repair of a single HO-induced DSB. First, the INO80 gene was deleted in a MATa strain that contains the GAL10-HO fusion gene and the HML $\alpha$ and HMRa homologous donors (JKM154 background) (Fig. 1A). In this case, the HO-induced DSB at the MAT locus is repaired by homologous recombination (HR), using homology at HML $\alpha$. Figure 1, B and C, shows the results of PCR-based assays that monitor the initial steps of HR, including DSB formation, DNA strand invasion at HML $\alpha$, and branch extension (White and Haber 1990).
After transfer of cells to galactose media, the kinetics of DSB formation are equivalent in the wild-type and ino $80 \Delta$ strains, with $\sim 75 \%$ of cells harboring a DSB within $60 \mathrm{~min}$ (Fig. 1B). A strand invasion PCR product can be detected in the wild-type strain by $2 \mathrm{~h}$, and formation of this product is linear over the first $3 \mathrm{~h}$ (Fig. 1C, left panel). In the ino80 $\Delta$ strain, there is a slight kinetic delay in strand invasion, although by $3 \mathrm{~h}$ after DSB formation the amount of strand invasion is equivalent between the wild-type and ino80 $\Delta$ strains. A similar delay in formation of the 500-bp branch extension product was observed in the ino80 mutant (Fig. 1C, right panel). In addition to monitoring these early events, formation of the final recombination product was also monitored by Southern analysis (White and Haber 1990). In this case as well, no dramatic decrease in product formation was observed in the absence of INO80 (Fig. 1D). Furthermore, both wild-type and ino80s cells yielded an equivalent number of colonies when the galactose-grown cells were subsequently plated on glucose media (data not shown). Thus, Ino80 appears to play only a minor role in DSB repair by $\mathrm{HR}$.

Nonhomologous end-joining of an HO-induced DSB was monitored in wild-type, ino80s, or ku70s strains that lacked the HML $\alpha$ and HMRa homologous donors (Fig. 2A, JKM179 "donorless" background; Paques and
A.

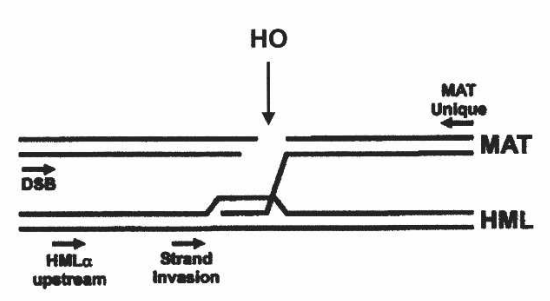

C.

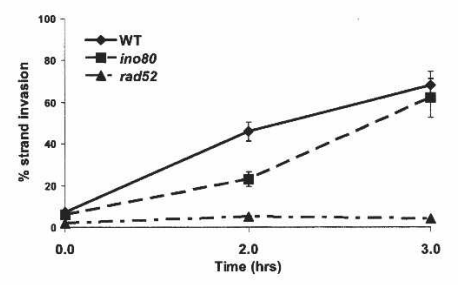

D.

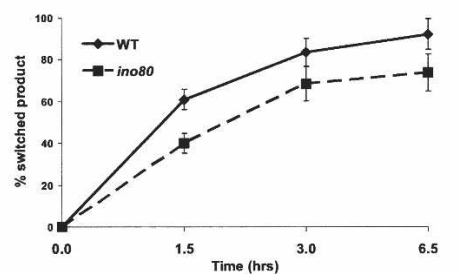

B.
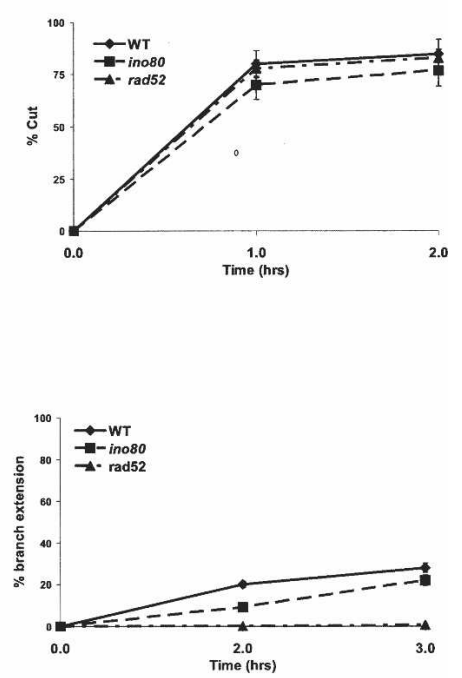

unique primer. Values of the strand invasion and branch extension PCR products were normalized to the percentage of DSB formation and the PHO5 ORF region. (D) Kinetics of gene conversion. Southern blot analysis for the switched product was conducted for wild-type (WT) and ino80 strains. Genomic DNA was isolated at the indicated time points, digested with StyI, and the values of the MAT $\alpha$ final product were quantified by PhosphorImager analysis and normalized to the initial DSB product and the MAT distal region.

Figure 1. Ino80 does not play a major role in recombinational repair. The indicated yeast strains were grown in raffinose media, galactose was added for $45 \mathrm{~min}$ to induce HO expression, glucose was then added to repress $\mathrm{HO}$ expression, and genomic DNA was isolated at the indicated time points and analyzed either by realtime PCR $(B, C)$ or by Southern blotting (D). (A) Schematic representation of chromosome III in yeast strain JKM154 (switching strain) bearing a $G A L-H O$ gene and containing the HML $\alpha$ homologous donor site. The arrow indicates the location of the HO cut site. (B) DSB occurs normally in the ino80 strain. Percent cut $(\%$ Cut) was measured as loss of the PCR product spanning the DSB (primers: MAT unique and $\mathrm{DSB}$ ) and normalized to the PHO5 ORF region. $(C)$ Kinetics of strand invasion and branch extension during switching. (Left) Strand invasion in the indicated strains was detected by PCR using the strand invasion primer that is $30 \mathrm{bp}$ upstream of the $\mathrm{HO}$ recognition site at $\mathrm{HML} \alpha$, and the MAT unique primer that is 428 bp downstream from the HO site at MAT. (Right) Branch extension was detected by PCR using the HML $\alpha$ upstream primer that is $470 \mathrm{bp}$ upstream of the HO
recognition site at HML $\alpha$ and the MAT recognition site at HML $\alpha$ and the MAT 
Papamichos-Chronakis et al.

A.

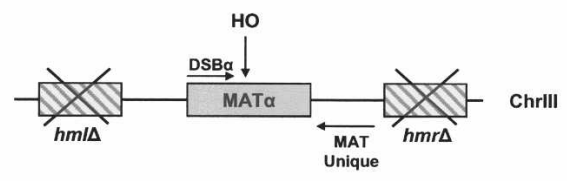

B.

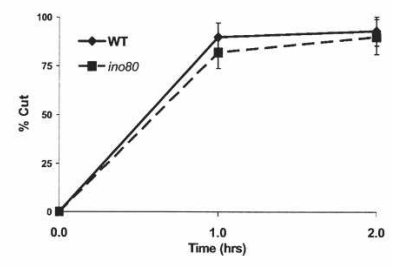

c.
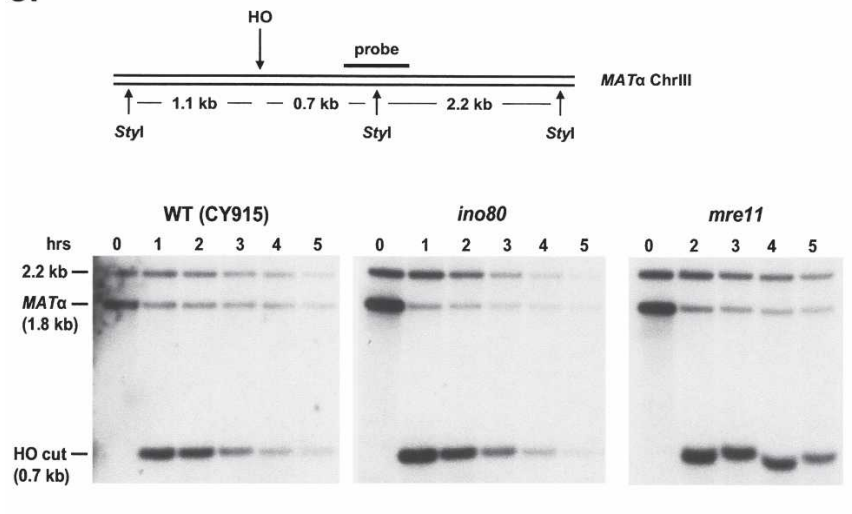

D.

E.

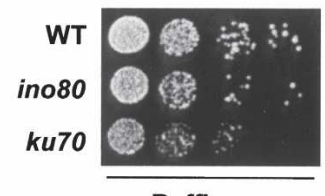

Raffinose

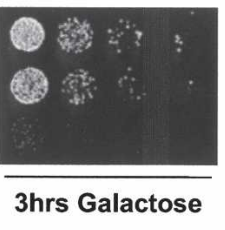

F.

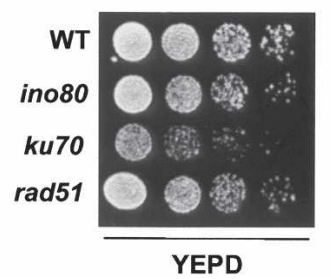

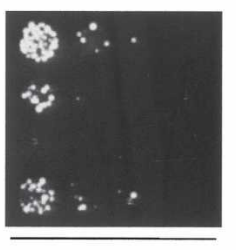

YEP+Galactose

Figure 2. Ino80 does not play a major role in nonhomologous end joining. (A) Schematic representation of chromosome III in yeast strain JKM179 (CY915, donorless) bearing a GAL-HO gene and deleted for the HML $\alpha$ and HMRa homologous donor sites. Arrows indicate the location of the HO cut site and of the two primers used to detect it. (B) DSB occurs normally in the ino80 "donorless" strain. GAL-HO was induced by addition of galactose in the indicated strains, and genomic DNA was isolated at the indicated time points and analyzed by real-time PCR. Percent cut (\% Cut) was measured as loss of the PCR product spanning the site of the break and normalized to the PHO5 ORF region. (C) Analysis of DSB formation and 5' $\rightarrow 3^{\prime}$ resection in wild-type (WT), ino80, and mre 11 strains. (Top) StyI restriction map at the MAT $\alpha$ locus. (Bottom) GAL-HO was induced by addition of galactose in the indicated strains, and genomic DNA was isolated at the indicated time points, digested with StyI and analyzed by Southern blot using the DNA probe illustrated at the top. The ssDNA generated by $5^{\prime} \rightarrow 3^{\prime}$ resection cannot be digested by StyI, leading to a gradual loss of the $0.7-$ and 2.2-kb bands. $(D)$ Quantification of the $5^{\prime} \rightarrow 3^{\prime}$ resection rate in the indicated strains. The values of the 0.7 -kb product from $C$ were quantified by PhosphorImager analysis and normalized to the URA3 locus and the 1.8-kb MAT $\alpha$ product at 0 h. The normalized 1.8 -kb MAT $\alpha$ product value was arbitrarily set as 100. (E) Ino80 is not necessary for error-free NHEJ. Mid-log cells from wild-type (WT), ino80, and ku70 donorless strains were grown in raffinose media and plated in 10-fold dilutions in YPD plates with or without prior addition of galactose for $3 \mathrm{~h}$ to induce $H O$ expression. Growth after galactose treatment requires NHEJ. Note that after $3 \mathrm{~h},>90 \%$ of cells harbor a DSB ( $B$; data not shown). ( $F)$ Analysis of error-prone NHEJ. Mid-log cells from the indicated strains were grown in raffinose media and plated in 10-fold dilutions on YPD or YP-Gal plates in order to induce constant $H O$ expression. Under these conditions the cells can repair the break and form colonies only by ligating the DSB and mutating the HO cut site.

Haber 1999|. In this case, the HO-induced DSB can only be repaired by NHEJ. Cells were grown in raffinose media, and then transferred to galactose media to induce formation of the DSB at the MAT locus. First, the kinetics of DSB formation was monitored by both a PCR- based assay (Fig. 2B) and by Southern blot (Fig. 2C). Both assays demonstrate equivalent kinetics of DSB formation in the wild-type and ino80s strains. We also monitored the exonucleolytic removal of the $5^{\prime} \rightarrow 3^{\prime}$ strand of DNA adjacent to the DSB (DNA resection), as a previous 
study showed that inactivation of the Arp8p subunit of the Ino80 complex led to slower kinetics of resection (van Attikum et al. 2004). DNA resection is commonly monitored by the time-dependent loss of a StyI restriction site located $0.7 \mathrm{~kb}$ from the $\mathrm{HO}$ cleavage site at MAT (see schematic in Fig. 2C). Thus, after formation of the HO-induced DSB, the loss of the 0.7- and 2.2-kb StyI fragments detected by Southern analysis provides a measurement of DNA resection kinetics. As expected (Ivanov et al. 1994; Lee et al. 1998; Tsubouchi and Ogawa 1998), inactivation of the Mre11 nuclease led to a noticeable defect in DNA resection (Fig. 2C,D), but no significant defect was observed in the ino80s strain (Fig. 2C,D).

To monitor whether Ino80p is required for completion of NHEJ, cells were grown in raffinose media, transferred to galactose media for $3 \mathrm{~h}$ to induce formation of the DSB at the MAT locus, and then serial dilutions of cells were plated on glucose plates to monitor successful NHEJ. As shown in Figure 2E, both the wild-type and ino80s strains showed equivalent growth on glucose media, indicating that inactivation of Ino80p does not cripple NHEJ of a chromosomal DNA DSB. As expected (Critchlow and Jackson 1998), inactivation of Ku70 crippled NHEJ, leading to poor growth on glucose (Fig. 2E).

An alternative strategy was also employed in which cells were grown in raffinose media and serial dilutions were plated directly onto galactose plates. Growth on galactose media requires an inefficient, error-prone NHEJ mechanism such that the HO recognition site is destroyed, preventing subsequent rounds of DSB formation. In this case only $1 \%-2 \%$ of the cells repair the DSB, while the majority of cells contain a persistent, unrepairable DSB. Robust growth on galactose media also requires that the cells remain viable in the presence of a persistent DSB until error-prone repair is accomplished. In this protocol, fewer colonies were observed for the ino80 $\Delta$ strain, although the defect is less severe than that

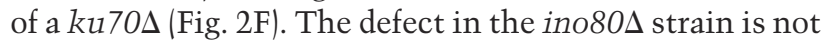
due to a defect in galactose fermentation, as colonies from both the ino80 $\mathrm{a}$ and wild-type plates were of similar size (Fig. 2F), and these colonies grew at identical rates when restreaked onto galactose media (data not shown). These results are consistent with a previous study (van Attikum et al. 2004). These data suggest that Ino80p may play a role specifically in the error-prone pathway of NHEJ. Alternatively, ino80 0 cells may lose viability more quickly in the presence of a persistent DNA DSB.

As an additional monitor of NHEJ efficiency, we also

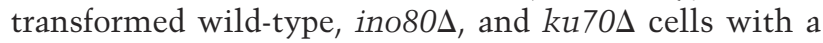
linearized CEN/ARS/URA3 plasmid. In this case, the number of $\mathrm{Ura}^{+}$transformants provides a quantitative measurement of NHEJ efficiency. In this case, the wildtype and ino80 $\Delta$ strains exhibited equivalent transformation efficiency, and the ku70s strain yielded transformants at only $2 \%$ of wild-type levels (Supplementary Fig. S2). Together, these data indicate that Ino80p does not have a major defect in the NHEJ pathway of DSB repair.
INO80 is required for DNA damage checkpoint adaptation

Since Ino80 appears to play only a minor role in the HR and NHEJ pathways, we investigated whether Ino80 is required for cell cycle checkpoint responses following formation of a persistent DSB. In GAL10-HO strains that lack homologous donors, continued growth in galactose media creates a persistent, unrepairable DSB that is sensed by the DNA damage checkpoint, leading to cell cycle arrest in the G2/M phase (Fig. 2F; note that only $1 \%-2 \%$ of cells repair the DSB by error-prone NHEJ; Toczyski et al. 1997). After 10-15 h of arrest, cells eventually escape from the checkpoint, enter mitosis with a broken chromosome, and form small microcolonies (Sandell and Zakian 1993; Toczyski et al. 1997).

To investigate roles for Ino80 in the DNA damage checkpoint, G1-phase wild-type and ino80d cells were micromanipulated to galactose plates to induce a persistent DSB, and cell cycle progression was monitored over time by following cell morphology. Within $8 \mathrm{~h}$, all of the wild-type and ino80 $\Delta$ cells had arrested as large budded cells characteristic of a G2/M arrest (Fig. 3C,D). By 20 h, nearly all of the wild-type cells had adapted to the checkpoint and formed small microcolonies of four to six cells (Fig. 3A,C). In contrast, the ino80 $\Delta$ cells were unable to adapt, remaining at the two-cell stage for at least $36 \mathrm{~h}$ and eventually losing viability (Fig. $3 \mathrm{~A}, \mathrm{D}$; data not shown). Furthermore, ino80 cells maintained high levels of Rad53p kinase activity 21-24 h after DSB formation, consistent with persistent checkpoint activation (Fig. 3B). FACS analysis of galactose-grown ino80A cells confirmed that the cells maintained a 2C DNA content (Supplementary Fig. S3). The inability of ino80 cells to form microcolonies represents a defect in checkpoint adaptation, as genetic inactivation of the checkpoint by deletion of the RAD17 or RAD9 genes allows both wildtype and ino80 $\Delta$ cells to form microcolonies on galactose media (Fig. 3E,F; Supplementary Fig. S4). Importantly, the adaptation defect of the ino80 8 strain was fully rescued by introduction of a CEN/ARS plasmid that contained INO80, but adaptation was not restored by expression of an ATPase-defective allele of INO80 (Ino80K737A) (Fig. 3G). Thus, checkpoint adaptation requires an intact Ino80 ATPase domain.

One possibility is that the defect in checkpoint adaptation is specific to a DSB at the MAT locus. In addition, it was possible that Ino80 is not specifically required for checkpoint adaptation, but that it is generally required for recovery from a checkpoint arrest. To test these ideas, we deleted the INO80 gene in a strain that harbors two defective copies of the LEU2 gene that are separated by $30 \mathrm{~kb}$ of genomic DNA (Fig. 3H). One LEU2 gene contains an $\mathrm{HO}$ recognition site, and galactose-dependent expression of HO leads to a single DSB that is repaired by the single-strand annealing (SSA) pathway (Vaze et al. 2002). This repair event utilizes homology within the other LEU2 fragment and generates an obligatory deletion of the $30 \mathrm{~kb}$ of intervening DNA. Wild-type and an ino80s derivative showed identical levels of 
Papamichos-Chronakis et al.

Figure 3. Ino80 is required for cell cycle A. checkpoint adaptation in response to a persistent DNA DSB. (A) Mid-log cells from wildtype (WT) and ino80 MAT $\alpha$ donorless strains were spread on galactose plates to induce GAL-HO. G1-phase (unbudded) cells were congregated on the plates by micromanipulation. Morphology and growth of the cells were observed and pictures were taken at $20 \mathrm{~h}$. (B) The Rad53p kinase is persistently active in the absence of INO80. In situ autophosphorylation assay was performed for TCA-extracted proteins from wild-type (WT) and ino80 donorless strains at the indicated times following DSB formation. Similar results were obtained from two independent experiments. $(C-F) \mathrm{G} 1$ cells $(0 \mathrm{~h})$ of the indicated donorless strains were micromanipulated onto galactose plates and their division was monitored at 8 and $24 \mathrm{~h}$. Microcolonies with a number of cells/buds that equals 2 represent the percentage of cells that have arrested at G2/M, whereas colonies with more than two cells/ buds indicate cells that adapt and resume division. (C) Wild type (WT). (D) ino80. (E) rad17. (F) rad17 ino80. (G) The ATPase domain of INO80 is required for adaptation. G1 cells $(0 \mathrm{~h})$ of the indicated donorless strains carrying either the empty vector, a plasmid expressing wild-type INO80 (pINO80), or an ATPase-defective allele of INO80 (pINO80K737A) were micromanipulated onto galactose plates and monitored at $24 \mathrm{~h}$. Analysis of cell number was performed as in $B$. (H) Ino80 is dispensable for SSA. (Top) Schematic representation of the SSA strain bearing a GAL$H O$ gene and deleted for the MAT, HML $\alpha$, and HMRa loci. The cells contain an HO cut site within the LEU2 gene (leu2::cs), and a fragment proximal to the cut site of the LEU2 gene has been inserted $30 \mathrm{~kb}$ distal. The galactose-induced DSB is repaired by SSA of the complementary LEU2 sequences, which are revealed as a result of the $5^{\prime} \rightarrow 3^{\prime}$ resection of the DSB ends. (Bottom) Mid-log cells from the indicated SSA strains were grown in raffinose media and plated in 10-fold dilutions onto YEPD and YEP-galactose plates. DSB occurred at equal levels in all strains as measured by quantitative PCR (data not shown; see also Fig. 4D, right panel). $(I, J) \mathrm{G} 1$ cells $(0 \mathrm{~h})$ of the indicated SSA strains were micromanipulated onto galactose plates and monitored at 8 and $24 \mathrm{~h}$ as in $B$. (I) rad52. (J) ino80 rad52.

colony formation on galactose media, indicating equivalent efficiencies of SSA (Fig. $3 \mathrm{H}$ ). Furthermore, since the slow repair of this DSB activates the cell cycle checkpoint (Vaze et al. 2002), the robust growth of the ino80s strain indicates that Ino80p is not required for recovery from checkpoint arrest following a successful repair event.

The SSA pathway requires Rad52p (Ivanov et al. 1996), and thus if a rad52 $\Delta$ is created in the SSA reporter strain and this strain is grown on galactose media, a persistent DSB is formed that activates the cell cycle checkpoint and then exhibits checkpoint adaptation after 10-15 h of arrest (Fig. 3I). However, an ino80 rad52 double mutant is unable to adapt to the persistent DSB at the SSA reporter locus (Fig. 3J). Thus, Ino80 is required for checkpoint adaptation irrespective of the site of the persistent DSB.

INO80 is required for maintenance of high levels of phosphorylated $\mathrm{H} 2 \mathrm{AX}$

How might an ATP-dependent chromatin remodeling enzyme control checkpoint adaptation? Recently, Shen and colleagues (Morrison et al. 2004) showed that phosphorylated histone H2AX is associated with Ino80 purified from DNA damaged cells. This interaction suggests 
that H2AX-phos might play a role in checkpoint adaptation. Therefore, we tested whether Ino80 might be required for accumulation of DNA damage-induced H2AX phosphorylation. First, we used Western blot analysis of bulk histones to assess the induction of H2AX-phos following exposure of cells to DNA damaging agents, MMS, campothecin (CPT), or phleomycin (Phleo). Whereas $\mathrm{H} 2 \mathrm{AX}$-phos is induced by all of these agents in wild-type cells, we observe a dramatic decrease in H2AX-phos in the ino80 $\Delta$ strain (eightfold decrease in MMS, threefold decrease in Phleo, and fivefold decrease in CPT) (Fig. 4A).

To confirm that the Western analyses accurately report on $\mathrm{H} 2 \mathrm{AX}$-phos levels in chromatin surrounding the DSB, chromatin immunoprecipitation (ChIP) analyses were performed in the "donorless" strains where $\mathrm{HO}$ creates a persistent DSB at the MAT locus. In this case, deletion of INO80 leads to an approximately twofold decrease in H2AX-phos detected by Western blot (Supplementary Fig. S5). ChIP analyses were also performed for these strains, using asynchronous cultures that rapidly accumulate at the G2/M boundary following formation of the DSB at the MAT locus. Following $20 \mathrm{~min}$ of $\mathrm{HO}$ expression, levels of $\mathrm{H} 2 \mathrm{AX}$-phos were increased 6.6- and 4.6-fold at 5 and $8 \mathrm{~kb}$ from the DSB in the wild-type strain, but $\mathrm{H} 2 \mathrm{AX}$-phos levels were only induced 2.6- and 3.4-fold in the ino80D (Fig. 4B, left panel). By $2 \mathrm{~h}$ of $\mathrm{HO}$ expression, H2AX-phos levels were induced 29- and 15fold in the wild-type strain, but H2AX-phos levels were only induced approximately fivefold in the ino80s strain (Fig. 4B, right panel). Thus, the results from the ChIP analyses correlate well with the defects observed by Western analyses, indicating that INO80 is required for fully induced levels of H2AX-phos.

\section{INO80 inhibits SWR1-dependent replacement of H2AX-phos with the Htz1p variant}

The decreased levels of H2AX-phos observed in the ino80 mutant suggest a simple model in which maintenance of this histone modification might be required for checkpoint adaptation. However, we find that a strain that lacks the H2AX phosphorylation site (hta1-S129A hta2-S129A) does not have a defect in adaptation, and in fact this strain appears to adapt with faster kinetics (data not shown). This was a surprising result, since the Ino80 complex is not recruited to a DNA DSB in the absence of H2AX-phos (Downs et al. 2004; van Attikum et al. 2004), and thus a simple model would predict that the hta1129A hta2-S129A strain would exhibit a phenotype identical to an ino80s.

Since ATP-dependent chromatin remodeling enzymes can catalyze the replacement of histone $\mathrm{H} 2 \mathrm{~A}$ variants (Mizuguchi et al. 2003; Kusch et al. 2004), we considered an alternative model in which loss of H2AX-phos in the ino80s mutant might be the result of a histone replacement event, involving either unphosphorylated H2AX or the Htzlp histone variant (Jackson and Gorovsky 2000). Recently, a Drosophila remodeling complex that harbors a Swr1p homolog was shown to replace damage-induced H2Av-Phos with H2Av (Kusch et al. 2004). In yeast, the Swr1 enzyme catalyzes replacement of H2AX with Htzlp at many sites, including centromeres and sites adjacent to heterochromatin (Krogan et al. 2003; Mizuguchi et al. 2003; Kober et al. 2004). Notably, the Htzlp variant has a distinct $\mathrm{C}$-terminal domain that cannot be phosphorylated by ATM-like kinases in response to DNA damage (Jackson and Gorovsky 2000).
A.
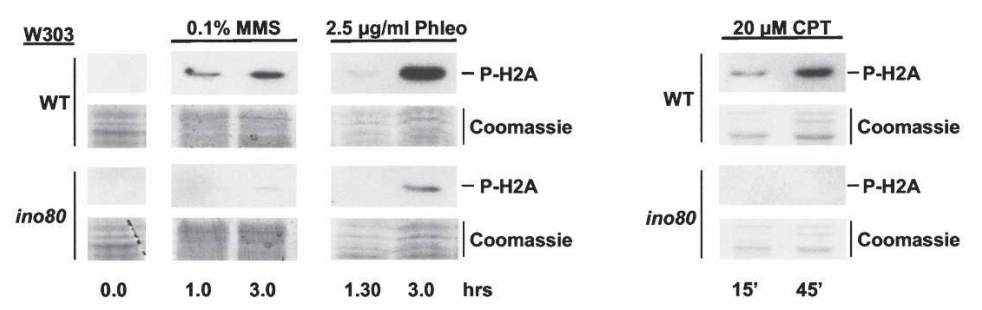

B.
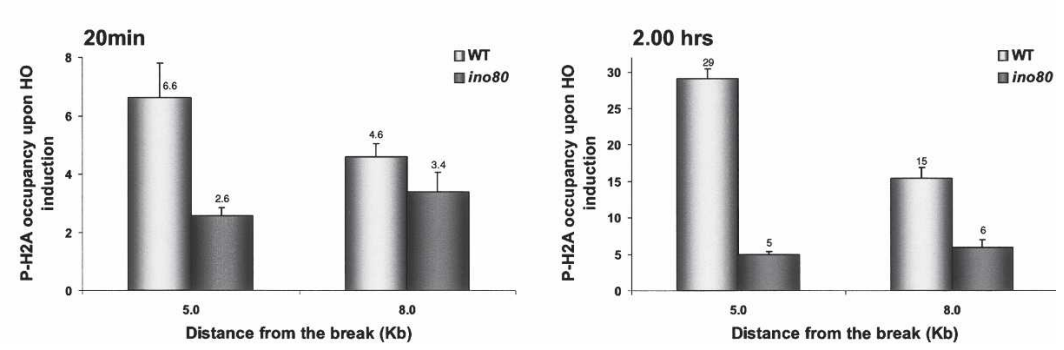
tion state of H2AX near the HO DSB is dependent on Ino80. ChIP analysis of H2AX-phos was conducted from asynchronous cultures of wild-type and ino 80 donorless strains grown in galactose for the indicated times. Occupancy at 5 and $8 \mathrm{~kb}$ next to the HO break was measured by quantitative PCR. Values reflect the fold enrichment of the tested DNA relative to the H2AX-phos levels before HO induction (time 0) after correction for the ratios of amplification achieved using input DNA.

Figure 4. Ino80 is required for high levels of DNA damage-induced H2AX phosphorylation. (A) Immunoblot analysis of H2AXphos in wild-type (WT) and ino80 mutant cells exposed to the indicated amounts of MMS, Phleo, and CPT for the indicated time. Acid-extracted proteins were separated by SDS page and analyzed with antiserum against the H2AX C-terminal phosphopeptide. In all Western blots, equal loading of the samples was confirmed by Ponceau-S staining of the membranes and verified by Coomassie staining of a parallel gel. The 10to $20-\mathrm{kDa}$ region of the respective Coomassie-stained gels is shown. We note that the levels of H2AX-phos are nearly equivalent in wild-type and ino80 cells if higher concentrations of MMS $(0.2 \%-0.3 \%)$ are used, consistent with a previous study (Morrison et al. 2004). Wild-type and ino80 samples treated with the same DNA damaging agent were electrophoresed in the same gel and processed together. $(B)$ Phosphoryla- 
To test the possibility that H2AX-phos was being replaced with histone Htzlp in an ino80s strain, we assessed Htzlp levels before and after formation of an unrepairable DSB using ChIP. Before formation of the DSB, Htzlp levels are equivalent in wild-type and ino80 $\Delta$ cells (Fig. 5A). Furthermore, Htzlp levels do not change significantly in wild-type cells following DSB formation (Fig. 5B-D). However, in the absence of Ino80p, Htzlp levels increase dramatically in chromatin adjacent to the DSB (Fig. 5B,D). In contrast, no changes in Htzlp levels were observed 30-50 kb from the DSB (Fig. 5C). Importantly, the increased levels of Htzlp found in the ino80s were still apparent even at $13 \mathrm{~h}$ after $\mathrm{HO}$ induction, a time point when wild-type cells undergo checkpoint adaptation (Fig. 5D). The aberrant incorporation of Htzlp is not detected in an ino80 hta1-S129A hta2-S129A strain, indicating that Htzlp incorporation in chromatin surrounding the DSB requires H2AX-phos (Fig. 5E). Notably, H2A-S129 is not required for Htzlp incorporation at sites distant from the DSB (e.g., PHO5, ASL1). Consis- tent with this observation, we observe a reciprocal relationship in the ino80s between levels of H2AX-phos and Htzlp when ChIP assays are performed from the same set of extracts (Fig. 5F).

To investigate whether the Swrlp remodeling enzyme is involved in H2AX-phos dynamics and checkpoint adaptation, we analyzed the phenotype of ino80s swr1s and ino80s htz1s double mutants (Fig. 6). Whereas Htzlaccumulates to high levels in chromatin adjacent to a DSB in the ino80 mutant, inactivation of Swrlp eliminates Htzlp and restores $\mathrm{H} 2 \mathrm{AX}$-phos in chromatin surrounding a DSB at the MAT locus (Fig. 6A,B). Likewise, removal of Htzlp or inactivation of Swrlp suppresses the defect in H2AX-phos accumulation observed in ino80s strains in response to CPT or MMS treatment (Fig. 6C,D). Furthermore, deletion of SWR1 or HTZ1 alleviates the checkpoint adaptation defect of the ino80D strain (Fig. 6E-G). Thus, these data suggest that Ino80 maintains high levels of H2AX-phos and activates checkpoint adaptation by antagonizing the ability of
A.

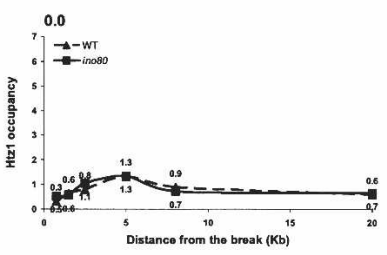

C.

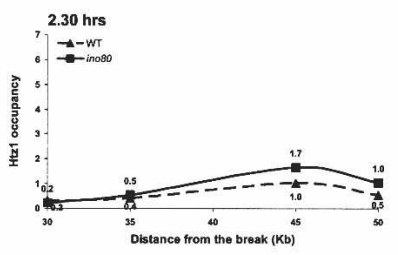

E.

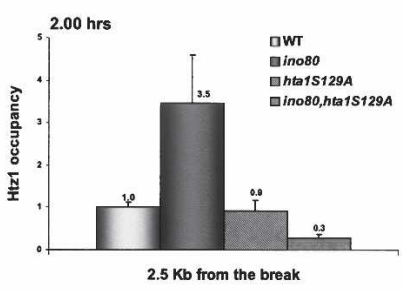

F.

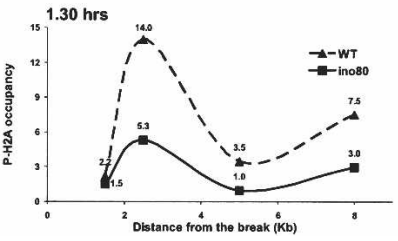

B.

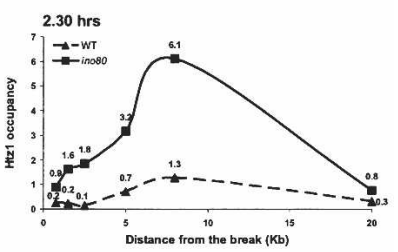

D.

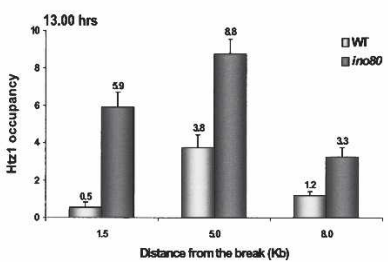

Figure 5. Ino80 regulates the Swr1-dependent deposition of Htzlp near a DSB. $(A-D)$ ChIP analysis of Htzl using polyclonal $\alpha-\mathrm{Htz} 1$ antiserum was conducted in the wild-type (WT) and ino80 donorless strains grown in galactose for the indicated times. Occupancy over the 20-kb region next to the HO break was measured by quantitative PCR with primers corresponding to regions $0.75,2.5,5.0,8.0$, and $20 \mathrm{~kb}$ distal to the DSB. In $C$, primers were used corresponding to regions $30,35,45$, and $50 \mathrm{~kb}$ distal to the DSB. Values reflect the fold enrichment of the tested DNA relative to an $\mathrm{rDNA}$ region $(A-C)$ or to a region of the $A S L 1$ ORF $(D)$ after correction for the ratios of amplification achieved using input DNA and normalized to the percentage of DSB formation in each strain. $(E)$ Recruitment of $\mathrm{Htzl}$ requires H2AX S129. ChIP analysis of Htzlp was conducted in wild-type, ino80A, hta1-S129A hta2-S129A, and ino80s hta1-S129A hta2-S129A donorless strains grown in galactose for $2 \mathrm{~h}$. Occupancy was measured as in $D .(F)$ H2AX-phos and Htzl occupy the region near the HO break in a reciprocal manner. ChIP analyses of H2AX-phos (left panel) and Htzl (right panel) were conducted in the wild-type (WT) and ino80 donorless strains grown in galactose for the indicated time. Occupancy over the 8 -kb region next to the HO break was measured by quantitative PCR with primers corresponding to regions 1.5, 2.5, 5.0, and $8.0 \mathrm{~kb}$ distal to the DSB. Analysis was performed as in $D$. 
A.

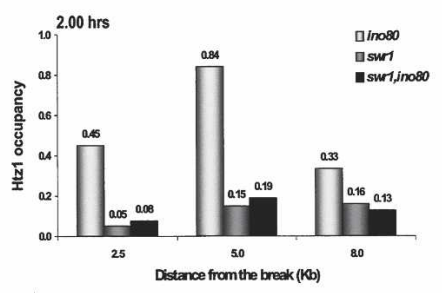

c.

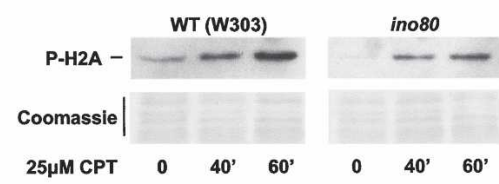

D.

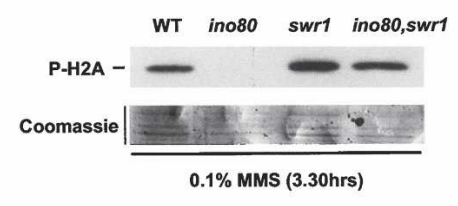

F.

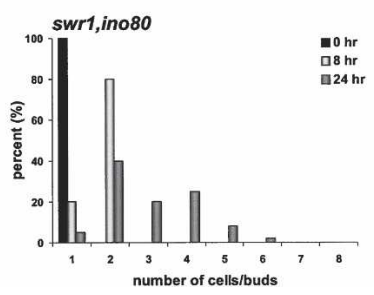

B.

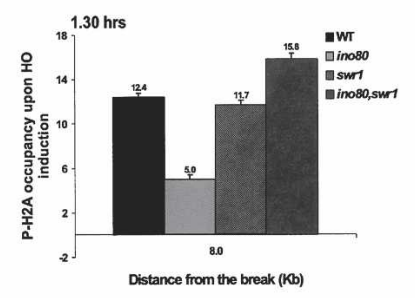

Figure 6. Inactivation of Swr1 restores H2AXphos and checkpoint adaptation in the absence of Ino80. (A) Recruitment of Htzl near the HO break is dependent on Swr1. ChIP analysis of Htzlp was conducted in ino80 and ino80 swr 1 donorless strains grown in galactose for $2.0 \mathrm{~h}$. Occupancy was measured by quantitative PCR using the same primers as in Figure 5D. Values are normalized to the percentage of DSB formation of each strain and reflect the ratio of the precipitated over the input DNA of each region. (B) Deletion of SWR1 restores the H2AX-phos levels near the HO DSB. ChIP analysis of H2AXphos was conducted in the indicated donorless strains grown in galactose for $1.30 \mathrm{~h}$. Occupancy was measured by quantitative PCR as in Figure 5D. $(C, D)$ H2AX-phos is recovered in the ino80 $h t z 1$ and ino80 swr1 double-mutant strains. $(C)$ Immunoblot analysis of H2AX-phos in wild-type (WT), ino80, htz1, and ino80 htz1 cells exposed to $25 \mu \mathrm{M}$ CPT for the indicated times. (D) Immunoblot analysis of H2AX-phos in wild-type (WT), ino80, swr1, and ino80 swr1 cells exposed to

G.

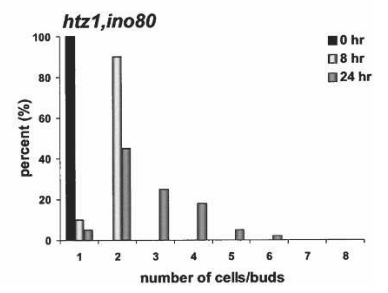
$0.1 \%$ MMS for the indicated time. Analysis was performed as in Figure 4A. $(E-G)$ Cell cycle checkpoint adaptation defect of the ino 80 strain is alleviated by either a $s w r 1$ or $h t z 1$ deletion. G1 cells $(0 \mathrm{~h})$ of ino80 $(E)$, swr1 ino80 $(F)$, and $h t z 1$ ino80 $(G)$ donorless strains were micromanipulated onto galactose plates and monitored at 8 and $24 \mathrm{~h}$. Analysis of cell number was performed as in Figure 3B.
Swrlp to replace H2AX-phos with Htzlp. These data also explain why the hta1-S129A hta2-S129A strain does not exhibit a defect in checkpoint adaptation-loss of H2AX-phos eliminates recruitment of Ino80 and also removes the substrate for histone replacement by the Swr1 enzyme.

\section{INO80 defines a new pathway required for checkpoint adaptation}

Previous studies have shown that the Ku70 end-binding protein and the Rad51p recombination protein are required for checkpoint adaptation (Lee et al. 1998, 2003). Furthermore, deletion of RAD52 suppresses the adaptation defect of rad51 mutants, but not that of yku70 mutants (Lee et al. 2003). Likewise, deletion of MRE11 suppresses the adaptation defect of yku70s, but not that of rad51s (Lee et al. 1998, 2003). These double mutant analyses provide evidence for at least two pathways that control the adaptation response. To investigate whether INO80 functions with either Ku70 or Rad51p, we cre-

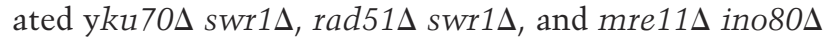

double mutants and assessed their ability to recover from checkpoint arrest. In contrast to the ino80 swr1 double mutant, deletion of SWR1 does not suppress the

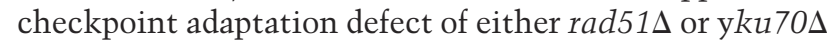
strains (Fig. 7C-F). Likewise, deletion of either RAD52 or $M R E 11$ does not suppress the checkpoint adaptation defect of an ino80 $\Delta$ strain (Figs. 3J, 7B). Thus, these genetic data support the view that INO80 and SWR1 function either downstream from Ku70 and Rad51p, or they define a novel pathway that regulates checkpoint adaptation.

\section{Discussion}

We have found that the Ino80 chromatin remodeling enzyme does not play a significant role in the repair of an HO-induced DNA DSB by either the homologous recombination or nonhomologous end-joining pathways. In contrast, we found that Ino80 is required for escape from a cell cycle checkpoint arrest due to a persistent, unrepairable DNA DSB. Furthermore, the defect in checkpoint adaptation that we observe in an ino80 correlated 
Papamichos-Chronakis et al.

A.

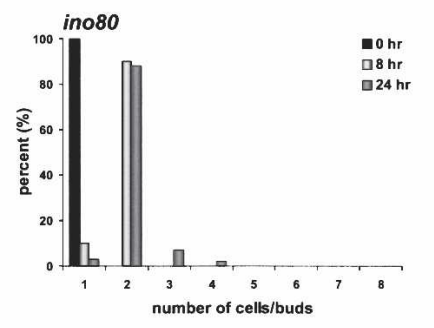

C.

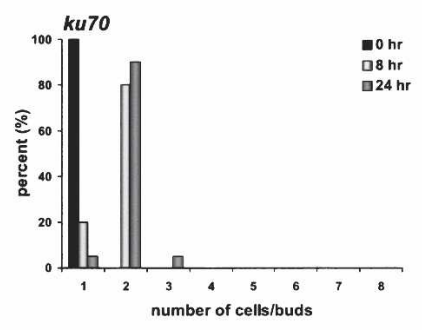

E.

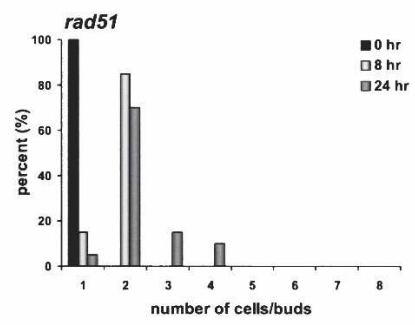

Figure 7. $(A-F)$ Ino80 defines a novel checkpoint adaptation pathway. G1 cells $(0 \mathrm{~h})$ of the indicated donorless strains were micromanipulated onto galactose plates and their division was monitored at 8 and $24 \mathrm{~h}$. Microcolonies with a number of cells/buds that equals 2 represent the percentage of cells that have arrested at G2/M, whereas colonies with more than two cells/buds indicate cells that adapt and resume division. $(A)$ ino80. (B) mre11 ino80. (C) ku70. (D) ino80 ku70. (E) rad51. (F) rad51 swr1. (G) Proposed model for the role of Ino80 and Swrl chromatin remodeling complexes during DNA damage. See the text for details.
G.

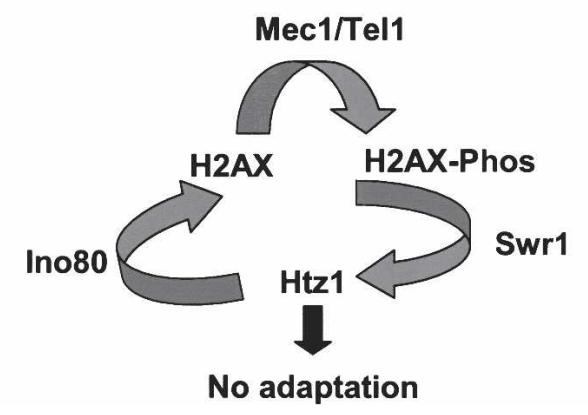

B.

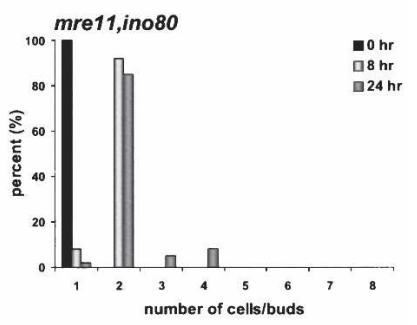

D.

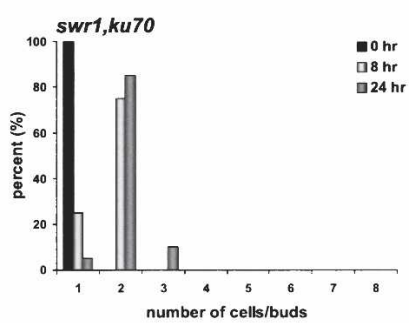

F.

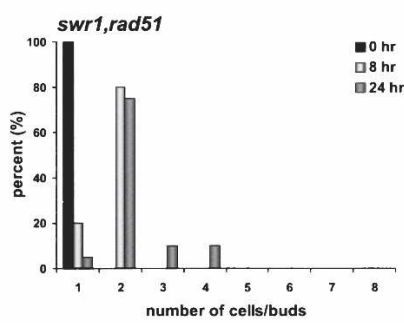

with low levels of H2AX phosphorylation and aberrant accumulation of the Htzlp histone variant in chromatin surrounding the DSB. Surprisingly, inactivation of the Swr1 remodeling enzyme restored H2AX phosphorylation, and checkpoint adaptation was restored by removal of either Swrl or the Htzlp histone variant. Furthermore, the novel accumulation of Htzlp required both Swrl and Ser 129 of H2AX, the residue that is phosphorylated in response to DNA damage. Thus, our data provide the first in vivo evidence that the Swrl remodeling enzyme can catalyze the replacement of H2AXphos with the Htzl variant. In addition, the data suggest that one key function for Ino80 in DNA repair is to antagonize the Htzlp replacement activity of the Swrl remodeling enzyme.
How might Ino80 antagonize Swrlp? One simple model is that Ino80 blocks the recruitment of Swrl to chromatin surrounding the DSB. Alternatively, we favor a model in which the ATP-dependent remodeling activity of Ino80 replaces Htzlp with H2AX, thereby restoring a substrate that can be phosphorylated by the checkpoint kinases, Meclp and Tellp (Fig. 7G). Consistent with this view, checkpoint adaptation requires the Ino80 ATPase domain. Thus, in this model, Ino80 and Swr1 catalyze a dynamic cycle of $\mathrm{H} 2 \mathrm{~A}$ variant exchange that not only influences the amount of H2AX-Phos but also determines the level of Htzlp incorporation. We envision that the relative amounts of these two distinct histone $\mathrm{H} 2 \mathrm{~A}$ variants might influence a decision-making process that controls whether to remove the checkpoint 
arrest and re-enter the cell cycle or to maintain arrest and eventually die. How these two histones modulate this signal to the checkpoint machinery is not known, but it seems likely that the mechanism will involve checkpoint signaling molecules that differentially interact with $\mathrm{H} 2 \mathrm{AX}$ or Htzlp.

\section{Ino80 and DNA repair mechanism}

A previous study suggested that the Ino80 chromatin remodeling complex contributes to DNA DSB repair by controlling the efficient exonucleolytic resection of the DNA ends, presumably by alterations in chromatin structure surrounding the DSB (van Attikum et al. 2004). Although our Southern analyses did not detect a significant defect in DNA resection in the absence of Ino80p, it seems plausible that our observed kinetic delay in DNA strand invasion is due to a defect in DNA resection in the absence of Ino80. This kinetic delay in HR may contribute to the DNA damage phenotypes of an ino80 mutant, but it seems unlikely that regulation of DNA resection is the primary role for Ino80 in DNA repair. Likewise, Osley and colleagues have reported that Ino80 contributes to a $\sim 50 \%$ decrease in nucleosome density in chromatin surrounding a DSB (Tsukuda et al. 2005). If more nucleosomes are indeed retained in the ino80s, then our data underestimate the loss of H2AX-phos. Likewise, nucleosome loss appears to be at least partially dependent on DNA resection surrounding the DSB (Tsukuda et al. 2005), but we find that Ino80 contributes to high levels of H2AX-phos even in G1-arrested cells where DNA resection is blocked (M. Papamichos-Chronakis and C.L. Peterson, unpubl.). Thus, effects of Ino80 on nucleosome loss or DNA resection do not contribute significantly to the functioning of Ino80 in checkpoint adaptation or maintenance of H2AX-phos.

\section{A complex role for H2AX phosphorylation during DSB repair}

Histone $\mathrm{H} 2 \mathrm{AX}$ is phosphorylated in response to a DNA DSB within a 1- to 2-Mb domain of chromatin in mammalian cells and a $\sim 100-\mathrm{kb}$ domain of chromatin in yeast. One role for $\mathrm{H} 2 \mathrm{AX}$-phos is to serve as a recruitment platform for DNA repair factors. For instance, studies in yeast have demonstrated that $\mathrm{H} 2 \mathrm{AX}$-phos recruits cohesin complex to the DSB region, which enhances recombinational repair of the DSB using the sister chromatid (Strom et al. 2004; Unal et al. 2004). Recent studies with mouse $\mathrm{H}_{2} \mathrm{AX}^{-/-}$cells are consistent with these results (Xie et al. 2004). It seems likely that Ino80 may also contribute to cohesin loading and sister chromatin recombination, as Ino80 is required for maintaining high levels of H2AX-phos. This potential defect in sister chromatid recombination may contribute to the sensitivity of an ino80 mutant to genotoxic agents.

Phosphorylation of H2AX in Schizosaccharmyces pombe also appears to stabilize the binding of checkpoint factors. In fission yeast, H2AX-phos stabilizes the binding of the Crb2p checkpoint factor adjacent to the DSB, and consequently, loss of H2AX-phos leads to premature release from cell cycle checkpoint arrest (Nakamura et al. 2004). This latter observation parallels our results indicating that hta1-S129A hta2-S129A cells perform checkpoint adaptation faster than wild-type cells.

In addition to promoting interactions with checkpoint factors and cohesin, H2AX-phos is also required for recruitment of the NuA4 histone acetyltransferase complex (Bird et al. 2002; Downs et al. 2004). Currently, it is not clear how NuA4 activity facilitates DNA repair, although a recent study indicates that histone acetylation by a Drosophila homolog of NuA4, Tip60, promotes exchange of a phosphorylated H2AX-like histone (Kusch et al. 2004). In contrast, our studies suggest that the recruitment of Ino80 by H2AX-phos may prevent the exchange of H2AX-phos. Thus, H2AX-phos may recruit factors that both promote or block its own turnover.

\section{Materials and methods}

Yeast strains and plasmids

Strains used in this study are derivatives of W303, JKM179 (CY915, Lee et al. 1998), and JKM154 (switching version of JKM179). The SSA strain (YMV2) is isogenic to YFP17, and has been described previously (Vaze et al. 2002). The INO80 gene was disrupted by replacing the first $0.9 \mathrm{~kb}$ of its coding region with either the KAN-MX6 gene that confers resistance to the drug G418, or with the TRP1 gene (Longtine et al. 1998). The KU70, RAD17, RAD9, MRE11, RAD52, RAD51, SWR1, and HTZ1 gene disruptions were generated as described (Longtine et al. 1998). The hta1-S129A hta2-S129A strains were constructed by sequential one-step insertion of a HisG-URA3-HisG cassette that contained the S129A codon substitution in the $5^{\prime}$ homologous primer sequences. Cells that had lost the URA3 cassette were selected by growth on 5-FOA. All of the disruptions were confirmed by PCR analysis and the constructed mutant strains exhibited the expected sensitivity on MMS, CPT, or $\mathrm{HU}$ plates. In addition, multiple independent transformants from INO80 strains disrupted with different marker genes were tested for adaptation, and all of them exhibited the same adaptation-defective phenotype.

The plasmid-borne copy of INO80 was constructed using a PCR fragment of INO80 containing the native promoter ( 600 $\mathrm{bp})$, the ORF, and the $3^{\prime}$ untranslated region ( 500 bp), which was cloned into the CEN/ARS vector, pRS416. A single amino acid substitution at the pINO80-416 was made by QuickChange mutagenesis (Stratagene) to create pINO80-K737A.

\section{$H O$ induction and DSB analysis}

Cells were grown at $30^{\circ} \mathrm{C}$ in YEP-raffinose $(2 \% \mathrm{w} / \mathrm{v})$ media, and galactose $(2 \% \mathrm{w} / \mathrm{v}$ final concentration) was added to mid-logphase cultures to induce HO. Aliquots were removed at the indicated time points and genomic DNA was extracted by a glass bead-phenol-sodium dodecyl sulfate protocol. For the homologous recombination assay, $\mathrm{HO}$ induction was repressed after $45 \mathrm{~min}$ by addition of glucose $(2 \% \mathrm{w} / \mathrm{v})$ to the medium.

For the PCR-based DSB and mating type switching analyses, quantitative real-time PCR was conducted using primers described in Wolner et al. (2003).

For the Southern blot analysis of MAT switching and $5^{\prime} \rightarrow 3^{\prime}$ resection at the MAT locus, genomic DNA was digested with 
StyI. The digested fragments were resolved on an agarose gel and transferred to hybridization membrane. The DNA probe hybridizes to a fragment that contains the first distal Styl restriction site (White and Haber 1990), and is common for the MATa and MAT $\alpha$ mating types. Quantification was performed by PhosphorImager analysis and DNA fragments in different samples were normalized either to the MAT distal region (for MAT switching) or to a URA3 fragment.

\section{Viability measurements}

For the nonhomologous end-joining assay, cells were grown until mid-log phase in YEP-raffinose, HO was induced for $3 \mathrm{~h}$, and then 10-fold serial dilutions of the uninduced and induced cultures were plated onto YEPD. Plates were incubated for 2-4 d. For the error-prone nonhomologous end-joining assay, cells were grown until mid-log phase in YEP-raffinose and 10-fold serial dilutions of the cultures were plated onto YEPD and YEP + Galactose plates. Plates were incubated for 2-4 d. For SSA, cells were grown until mid-log phase in YEP-raffinose and 10 -fold serial dilutions of cultures were plated onto YEPD and YEP-galactose. Plates were incubated for 2-4 d.

\section{Microscopic analysis of cell morphology}

Mid-log-phase cells grown in YEP-raffinose were spread onto agar plates containing YEP-galactose media. G1 (unbudded) cells were congregated on the plate by micromanipulation. Cell growth and division were monitored and microcolony formation was recorded by microscopic photography.

\section{Plasmid repair assay}

Cells were transformed with $200 \mathrm{ng}$ of EcoRI-linearized or mock-digested pRS416. Transformations were plated onto selective medium (SC-Ura) and colonies were counted after $3 \mathrm{~d}$ at $30^{\circ} \mathrm{C}$.

\section{ChIP}

ChIPs were performed as described by Papamichos-Chronakis et al. (2002) using commercially available polyclonal antibody raised against the phosphorylated form of serine residue 129 of histone $\mathrm{H} 2 \mathrm{~A}(\mathrm{AbCam})$ and polyclonal antibody specific for the histone Htzlp (AbCam). The recovered DNA was subjected to quantitative real-time PCR. All ChIPs and PCR reactions were performed at least twice (Papamichos-Chronakis et al. 2002), and the variation between experiments was $10 \%-25 \%$. The primers at the donorless strains covering the region next to the HO cut site are proximal to the telomere. Primers used in the PCR reactions are available upon request.

\section{Protein analysis and in situ autophosphorylation assay}

Proteins from yeast whole-cell extracts were extracted with sulfuric acid as described (Redon et al. 2003). Proteins were resolved on $15 \%$ SDS page and either analyzed by immunoblotting using a chicken antibody to H2AX-phos (Aves laboratories) following standard procedures, or stained with Coomassie. The specificity of the H2AX-phos antibody was verified using extracts from CPT-treated wild-type and hta1-S129A hta2-S129A strains (Supplementary Fig. S6). Quantification of H2AX-phos signal was conducted using the Scion Image Analysis program and normalized to the respective Coomassie staining. The In Situ Autophosphorylation assay was conducted as described in Pellicioli et al. (2001).

\section{Acknowledgments}

We thank Branden Wolner and Nastaran Azizian for materials and helpful discussions, Jim Haber for providing invaluable yeast strains, and Anthony Imbalzano for assistance with the real-time PCR analyses. This work was supported by a grant (GM54096) from the National Institutes of Health.

\section{References}

Bassing, C.H., Chua, K.F., Sekiguchi, J., Suh, H., Whitlow, S.R., Fleming, J.C., Monroe, B.C., Ciccone, D.N., Yan, C., Vlasakova, K., et al. 2002. Increased ionizing radiation sensitivity and genomic instability in the absence of histone H2AX. Proc. Natl. Acad. Sci. 99: 8173-8178.

Bird, A.W., Yu, D.Y., Pray-Grant, M.G., Qiu, Q., Harmon, K.E., Megee, P.C., Grant, P.A., Smith, M.M., and Christman, M.F. 2002. Acetylation of histone $\mathrm{H} 4$ by Esal is required for DNA double-strand break repair. Nature 419: 411-415.

Celeste, A., Petersen, S., Romanienko, P.J., Fernandez-Capetillo, O., Chen, H.T., Sedelnikova, O.A., Reina-San-Martin, B., Coppola, V., Meffre, E., Difilippantonio, M.J., et al. 2002. Genomic instability in mice lacking histone H2AX. Science 296: 922-927.

Celeste, A., Difilippantonio, S., Difilippantonio, M.J., Fernandez-Capetillo, O., Pilch, D.R., Sedelnikova, O.A., Eckhaus, M., Ried, T., Bonner, W.M., and Nussenzweig, A. 2003a. H2AX haploinsufficiency modifies genomic stability and tumor susceptibility. Cell 114: 371-383.

Celeste, A., Fernandez-Capetillo, O., Kruhlak, M.J., Pilch, D.R., Staudt, D.W., Lee, A., Bonner, R.F., Bonner, W.M., and Nussenzweig, A. 2003b. Histone H2AX phosphorylation is dispensable for the initial recognition of DNA breaks. Nat. Cell Biol. 5: 675-679.

Critchlow, S.E. and Jackson, S.P. 1998. DNA end-joining: From yeast to man. Trends Biochem. Sci. 23: 394-398.

Downs, J.A., Lowndes, N.F., and Jackson, S.P. 2000. A role for Saccharomyces cerevisiae histone H2A in DNA repair. $\mathrm{Na}$ ture 408: 1001-1004.

Downs, J.A., Allard, S., Jobin-Robitaille, O., Javaheri, A., Auger, A., Bouchard, N., Kron, S.J., Jackson, S.P., and Cote, J. 2004. Binding of chromatin-modifying activities to phosphorylated histone H2A at DNA damage sites. Mol. Cell 16: 979990.

Ebbert, R., Birkmann, A., and Schuller, H.J. 1999. The product of the SNF2/SWI2 paralogue INO80 of Saccharomyces cerevisiae required for efficient expression of various eyast structural genes is part of a high-molecular-weight protein complex. Mol. Microbiol. 32: 741-751.

Ivanov, E.L., Sugawara, N., White, C.I., Fabre, F., and Haber, J.E. 1994. Mutations in XRS2 and RAD50 delay but do not prevent mating-type switching in Saccharomyces cerevisiae. Mol. Cell. Biol. 14: 3414-3425.

Ivanov, E.L., Sugawara, N., Fishman-Lobell, J., and Haber, J.E. 1996. Genetic requirements for the single-strand annealing pathway of double-strand break repair in Saccharomyces cerevisiae. Genetics 142: 693-704.

Jackson, J.D. and Gorovsky, M.A. 2000. Histone H2A.Z has a conserved function that is distinct from that of the major H2A sequence variants. Nucleic Acids Res. 28: 3811-3816.

Khanna, K.K. and Jackson, S.P. 2001. DNA double-strand breaks: Signaling, repair and the cancer connection. Nat. Genet. 27: 247-254.

Kober, M.S., Venkatasubrahmanyam, S., Meneghini, M.D., Gin, J.W., Jennings, J.L., Link, A.J., Madhani, H.D., and Rine, J. 2004. A protein complex containing the conserved Swi2/ 
Snf2-related ATPase Swrlp deposits histone variant H2A.Z into euchromatin. PLOS Biol. 2: E131.

Krogan, N.J., Keogh, M.-C., Datta, N., Sawa, C., Ryan, O.W., Ding, H., Haw, R.A., Pootoolal, J., Tong, A., Canadien, V., et al. 2003. A Snf2 family ATPase complex required for recruitment of the histone H2A variant Htz1. Mol. Cell 12: 15651576.

Kusch, T., Florens, L., Macdonald, W.H., Swanson, S.K., Glaser, R.L., Yates III, J.R., Abmayr, S.M., Washburn, M.P., and Workman, J.L. 2004. Acetylation by Tip60 is required for selective histone variant exchange at DNA lesions. Science 306: 2084-2087.

Lee, S.E., Moore, J.K., Holmes, A., Umezu, K., Kolodner, R.D., and Haber, J.E. 1998. Saccharomyces Ku70, Mre11/Rad50, and RPA proteins regulate adaptation to G2/M arrest after DNA damage. Cell 94: 399-409.

Lee, S.E., Pellicioli, A., Vaze, M.B., Sugawara, N., Malkova, A., Foiani, M., and Haber, J.E. 2003. Yeast Rad52 and Rad51 recombination proteins define a second pathway of DNA damage assessment in response to a single double-strand break. Mol. Cell. Biol. 23: 8913-8923.

Longtine, M.S., McKenzie III, A., Demarini, D.J., Shah, N.G., Wach, A., Brachat, A., Philippsen, P., and Pringle, J.R. 1998. Additional modules for versatile and economical PCR-based gene deletion and modification in Saccharomyces cerevisiae. Yeast 14: 953-961.

Lupardus, P.J. and Cimprich, K.A. 2005. Checkpoint adaptation: Molecular mechanisms uncovered. Cell 17: 555-559.

Lydall, D. and Weinert, T. 1995. Yeast checkpoint genes in DNA damage processing: Implications for repair and arrest. Science 270: 1488-1491.

Mizuguchi, G., Shen, X., Landry, J., Wu, W.H., Sen, S., and Wu, C. 2003. ATP-driven exchange of histone H2AZ variant catalyzed by SWR 1 chromatin remodeling complex. Science 303: 343-348.

Morrison, A.J., Highland, J., Krogan, M., Arbel-edi, A., Greenblatt, J.F., Haber, J.E., and Shen, X. 2004. INO80 and $\gamma$-H2AX interaction links ATP-dependent chromatin remodeling to DNA damage repair. Cell 119: 767-775.

Nakamura, T.M., Du, L.L., Redon, C., and Russell, P. 2004. Histone H2A phosphorylation controls $\mathrm{Crb} 2$ recruitment at DNA breaks, maintains checkpoint arrest, and influences DNA repair in fission yeast. Mol. Cell. Biol. 24: 6215-6230.

Papamichos-Chronakis, M., Petrakis, T., Ktistaki, E., Topalidou, I., and Tzamarias, D. 2002. Cti6, a PHD domain protein, bridges the Cyc8-Tupl corepressor and the SAGA coactivator to overcome repression at GAL1. Mol. Cell 9: 1297-1305.

Paques, F. and Haber, J.E. 1999. Multiple pathways of recombination induced by double-strand breaks in Saccharomyces cerevisiae. Microbiol. Mol. Biol. Rev. 63: 349-404.

Pellicioli, A., Lee, S.E., Lucca, C., Foiani, M., and Haber, J.E. 2001. Regulation of Saccharomyces Rad53 checkpoint kinase during adaptation from a DNA damage-induced G2/M arrest. Mol. Cell 7: 293-300.

Peterson, C.L. and Cote, J. 2004. Cellular machineries for chromosomal DNA repair. Genes \& Dev. 18: 602-616.

Redon, C., Pilch, D.R., Rogakou, E.P., Orr, A.H., Lowndes, N.F., and Bonner, W.M. 2003. Yeast histone 2A serine 129 is essential for the efficient repair of checkpoint-blind DNA damage. EMBO Rep. 4: 678-684.

Rogakou, E.P., Boon, C., Redon, C., and Bonner, W.M. 1999. Megabase chromatin domains involved in DNA doublestrand breaks in vivo. J. Cell Biol. 146: 905-916.

Sandell, L.L. and Zakian, V.A. 1993. Loss of a yeast telomere: Arrest, recovery, and chromosome loss. Cell 75: 729-739.
Shen, X., Mizuguchi, G., Hamiche, A., and Wu, C. 2000. A chromatin remodelling complex involved in transcription and DNA processing. Nature 406: 541-544.

Shroff, R., Arbel-Eden, A., Pilch, D., Ira, G., Bonner, W.M., Petrini, J.H., Haber, J.E., and Lichten, M. 2004. Distribution and dynamics of chromatin modification induced by a defined DNA double-strand break. Curr. Biol. 14: 1703-1711.

Strom, L., Lindroos, H.B., Shirahige, K., and Sjogren, C. 2004. Postreplicative recruitment of cohesin to double-strand breaks is required for DNA repair. Mol. Cell 16: 1003-1015.

Symington, L.S. 2002. Role of RAD52 epistasis group genes in homologous recombination and double-strand break repair. Microbiol. Mol. Biol. Rev. 66: 630-670.

Toczyski, D.P., Galgoczy, D.J., and Hartwell, L.H. 1997. CDC5 and CKII control adaptation to the yeast DNA damage checkpoint. Cell 90: 1097-1106.

Tsubouchi, H. and Ogawa, H. 1998. A novel mrel1 mutation impairs processing of double-strand breaks of DNA during mitosis and meiosis. Mol. Cell 18: 260-268.

Tsukuda, T., Fleming, A.B., Nickoloff, J.A., and Osley, M.A. 2005. Chromatin remodelling at a DNA double-strand break site in Saccharomyces cerevisiae. Nature 438: 379-383.

Unal, E., Arbel-Eden, A., Sattler, U., Shroff, R., Lichten, M., Haber, J.E., and Koshland, D. 2004. DNA damage response pathway uses histone modification to assemble a doublestrand break-specific cohesion domain. Mol. Cell 16: 9911002 .

van Attikum, H., Fritsch, O., Hohn, B., and Gasser, S.M. 2004. Recruitment of the INO80 complex by H2A phosphorylation links ATP-dependent chromatin remodeling with DNA double-strand break repair. Cell 119: 777-788.

Vaze, M.B., Pellicioli, A., Lee, S.E., Ira, G., Liberi, G., ArbelEden, A., Foiani, M., and Haber, J.E. 2002. Recovery from checkpoint-mediated arrest after repair of a double-strand break requires Srs2 helicase. Mol. Cell 10: 373-385.

White, C.I. and Haber, J.E. 1990. Intermediates of recombination during mating type switching in Saccharomyces cerevisiae. EMBO J. 9: 663-673.

Wolner, B., van Komen, S., Sung, P., and Peterson, C.L. 2003. Recruitment of the recombinational repair machinery to a DNA double-strand break in yeast. Mol. Cell 12: 221-232.

Xie, A., Puget, N., Shim, I., Odate, S., Jarzyna, I., Bassing, C.H., Alt, F.W., and Scully, R. 2004. Control of sister chromatid recombination by histone H2AX. Mol. Cell 16: 1017-1025.

Yoo, H.Y., Kumagai, A., Shevchenko, A., Shevchenko, A., and Dunphy, W.G. 2005. Adaptation of a DNA replication checkpoint response depends upon inactivation of Claspin by the Polo-like kinase. Cell 17: 575-588. 


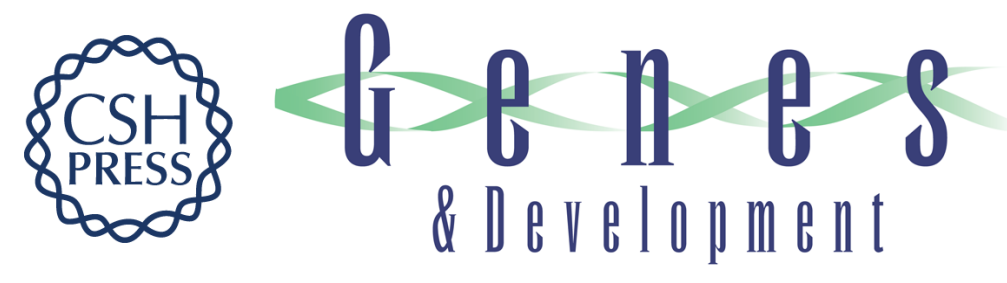

\section{Interplay between Ino80 and Swr1 chromatin remodeling enzymes regulates cell cycle checkpoint adaptationin response to DNA damage}

Manolis Papamichos-Chronakis, Jocelyn E. Krebs and Craig L. Peterson

Genes Dev. 2006, 20:

Access the most recent version at doi:10.1101/gad.1440206

Supplemental http://genesdev.cshlp.org/content/suppl/2006/09/05/20.17.2437.DC1
Material

References This article cites 44 articles, 12 of which can be accessed free at:

http://genesdev.cshlp.org/content/20/17/2437.full.html\#ref-list-1

License

Email Alerting Receive free email alerts when new articles cite this article - sign up in the box at the top

Service

right corner of the article or click here.

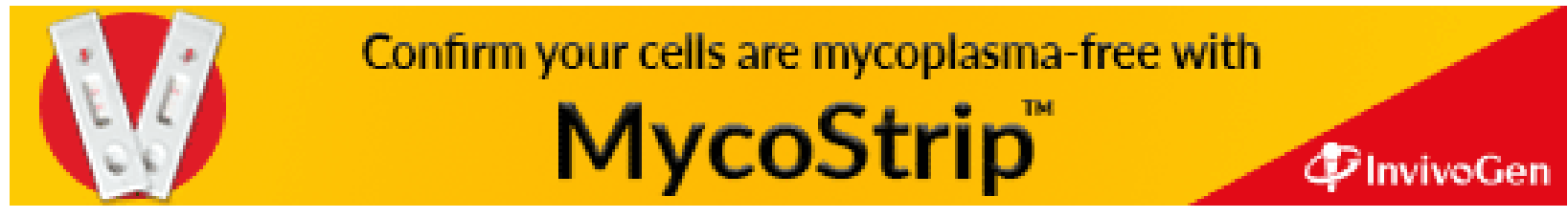

